Journal of Magnetism and Magnetic Materials 3 (1976) 4-6

(c) North-Holland Publishing Company

\title{
THE TEMPERATURE DEPENDENCE OF THE SPIN POLARIZATION OF FIELD EMITTED ELECTRONS FROM A W-EUS-VACUUM JUNCTION
}

\author{
G. BAUM, E. KISKER, A.H. MAHAN and K. SCHRÖDER \\ Universität Bielefeld, Fakultät für Physik, 48 Bielefeld 1, Viktoriastrasse 44, Federal Republic of Germany
}

\begin{abstract}
We have measured the temperature dependence $P(T)$ of the electron spin polarization of field emitted electrons from a W-EuS-vacuum junction. The shapes of the $P(T)$ curves depend strongly on the annealing temperature of the EuS layer. Annealed at some temperature between $300^{\circ} \mathrm{C}$ and $600^{\circ} \mathrm{C}$ the polarization drops to zero at about $16 \pm 2 \mathrm{~K}$.
\end{abstract}

\section{Introduction}

For development of a polarized electron source suitable for atomic physics scattering experiments we studied field emission from tungsten tips coated with EuS. It was discovered by N. Müller et al. [1] that such electrons can be spin polarized to a high degree. We extended the experimental conditions of Muiller et al. to explore the most favorable circumstances under which highly polarized electrons can be obtained. In particular, we made the tip temperature continuously variable from $10 \mathrm{~K}$ up to room temperature. We report in this work temperature dependences of the electron spin polarization obtained with different annealing conditions of the EuS layer.

\section{Apparatus}

The apparatus consists of three parts: a) field emission system, b) Wien filter, c) Mott scattering polarization detector. The field emission takes place in an ultrahigh vacuum chamber where a pressure lower than $10^{-10}$ torr is maintained during field emission studies. The EuS is evaporated from an oven, which for this purpose can be moved onto the electron optical axis, with the orifice $6 \mathrm{~cm}$ in front of the field emission tip. The oven is heated to about $2000^{\circ} \mathrm{C}$ by electron bombardment. The evaporation time is 2 minutes, and during this process the pressure rises to about $10^{-8}$ torr.

The tip is mounted on a cold finger working on the dynamical flow principle [2]. The tip temperature can be controlled between $10 \mathrm{~K}$ and room temperature by regulating the flow of the liquid helium coolant. Higher temperatures, which are used for the annealing process of the EuS layer, can be achieved by resistance heating of the tip-support filament. Above $600^{\circ} \mathrm{C}$ the thermal glow can be viewed through a telescope; lower temperatures are qualitatively controlled by recording the heating current. An axial static magnetic field up to $5 \mathrm{kG}$ can be produced in the tip region by an electromagnet. The field emission pattern can be observed on a fluorescent screen $15 \mathrm{~cm}$ away from the tip. The electron beam can be moved across the screen by electrostatic deflection in order to transmit a selected part of the field emission pattern through the central probe hole. The emerging beam leaves the ultrahigh vacuum chamber through a differential pumping hole. The beam then traverses a Wien filter (crossed electric and magnetic fields) capable of turning the polarization vector transverse to the beam direction, as only transverse polarization can be determined by Mott scattering. The targets for Mott scattering are thin gold and aluminium foils which can be placed successively on the electron beam axis. The asymmetry of the backscattered electrons is measured with two pairs of surface-barrier detectors which are positioned at a scattering angle of $120^{\circ}$ and azimuthal angles of $0^{\circ}, 180^{\circ}$ and $90^{\circ}, 270^{\circ}$ respectively. Simultaneous measurement of two of the three components of the polarization vector is therefore possible. The third component is made to vanish by proper setting of the Wien filter. 


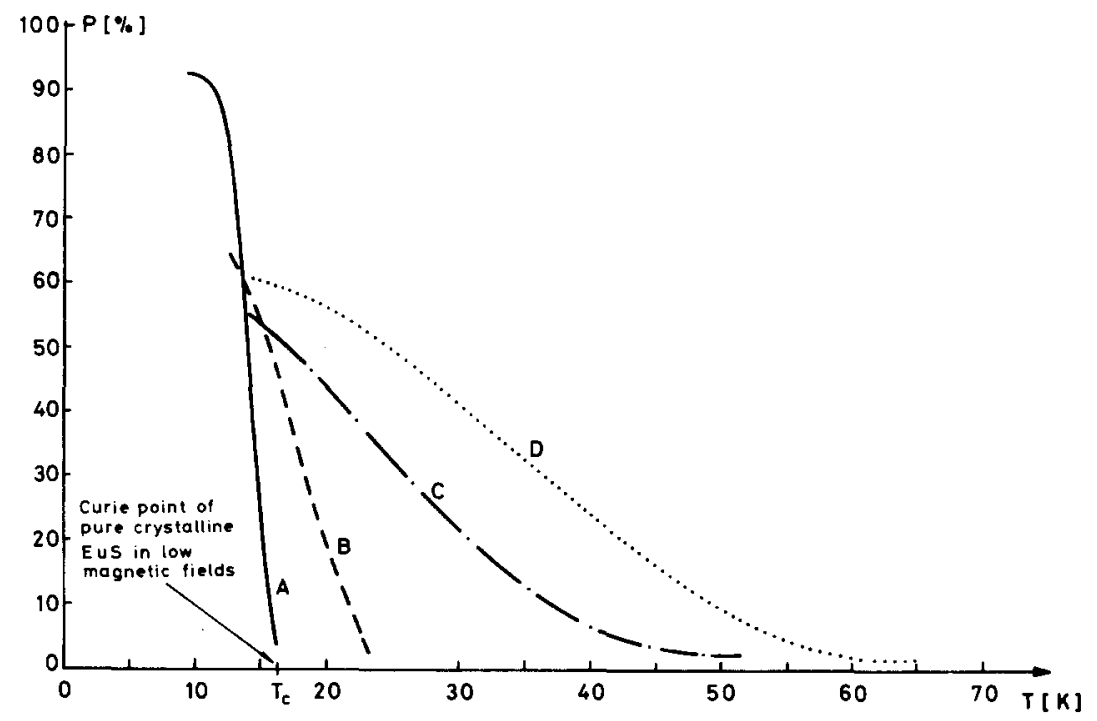

Fig. 1. The temperature dependence of the electron spin polarization of field emitted electrons from EuS coated tungsten tips for different annealing temperatures. For curves $A$ and $B$ the total polarization value is shown. The error is about \pm 0.07 . For the earlier curves $C$ and $D$ one component of the polarization vector has not been determined and the total polarization may be higher. The temperature scale is uncertain to about $\pm 2 \mathrm{~K}$. Curves $A$ and $B$ were measured at magnetic fields of $250 \mathrm{G}$, curves $C$ and $D$ at about $3 \mathrm{kG}$.

\section{Results}

The measured dependences of the polarization value upon tip temperature are shown in fig. 1. Curve $A$ was obtained after annealing the tip at some temperature between $300^{\circ} \mathrm{C}$ and $600^{\circ} \mathrm{C}$. It shows a very sharp increase in polarization below the Curie point of pure crystalline EuS, with emission in the 112 directions. As we have shown in ref. [3] , this is accompanied by a drastic increase of the toal emission current. This increase in current does not occur if the freshly evaporated EuS deposit is either not annealed, or annealed at too low a temperature; it disappears at higher annealing temperatures. We did not measure $P(T)$ curves for unannealed EuS layers because the emission is very unstable. In curve $\mathrm{B}$, obtained after several annealing cycles at about $600^{\circ} \mathrm{C}$, only one point of the emission pattern shows the current increase, as seen on the fluorescent screen, and the polarization approaches zero at higher temperatures. Curves $C$ and $D$ are two different sets of data showing the temperature dependence of the electron polarization of coatings which have been annealed at approximately $800^{\circ} \mathrm{C}$. No current increase was observed, and the extension of appreciable polarization to higher temperatures is even more dras- tic. The polarization values in curves $\mathrm{C}$ and $\mathrm{D}$ may be even higher because one transverse component of the polarization vector has not been measured.

\section{Discussion}

Our explanation of the measured temperature dependences of the spin polarization is based on the results of Bayer and Zinn [4], who measured the temperature dependence of the saturation magnetization of EuS layers. They show that moderate annealing of the freshly evaporated EuS film results in a Curie point of $16.5 \mathrm{~K}$, the Curie point of pure crystalline EuS. The temperature dependence of the saturation magnetization of this species and of our spin polarization, shown in curve $A$, are very similar. We therefore believe that curve $A$ represents the temperature dependence of the polarization which is characteristic of electron emission from pure crystalline EuS. The polarization dependences of the other curves (B, C, D of fig. 1), which look similar to magnetization curves of Bayer and Zinn obtained from EuS layers with disturbed crystal structures, could be attributed to a partial decomposition of the EuS caused by higher annealing temperatures. 


\section{Acknowledgment}

We thank Dipl.-Phys. N. Müller for helpful discussions. We are grateful to Prof. W. Raith for encouraging support and valuable assistance. This work has been supported by the University of Bielefeld (Project No. 2854).

\section{References}

[1] N. Müller, W. Eckstein, W. Heiland and W. Zinn, Phys. Rev. Letters 29 (1972) 1651

[2] D.A. Reed and W.R. Graham, Rev. Sci. Instr. 43 (1972) 1365.

[3] E. Kisker, G. Baum, A.H. Mahan, W. Raith and K. Schröder, to be published.

[4] E. Bayer and W. Zinn, Z. angew. Physik 32 (1971) 83. 